\title{
Revisión del proceso de la degradación en los aceites lubricantes en motores de gas natural comprimido y diesel
}

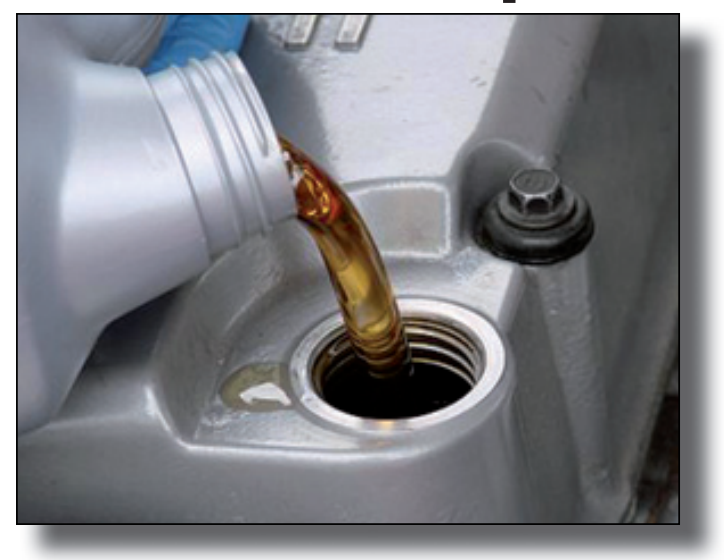

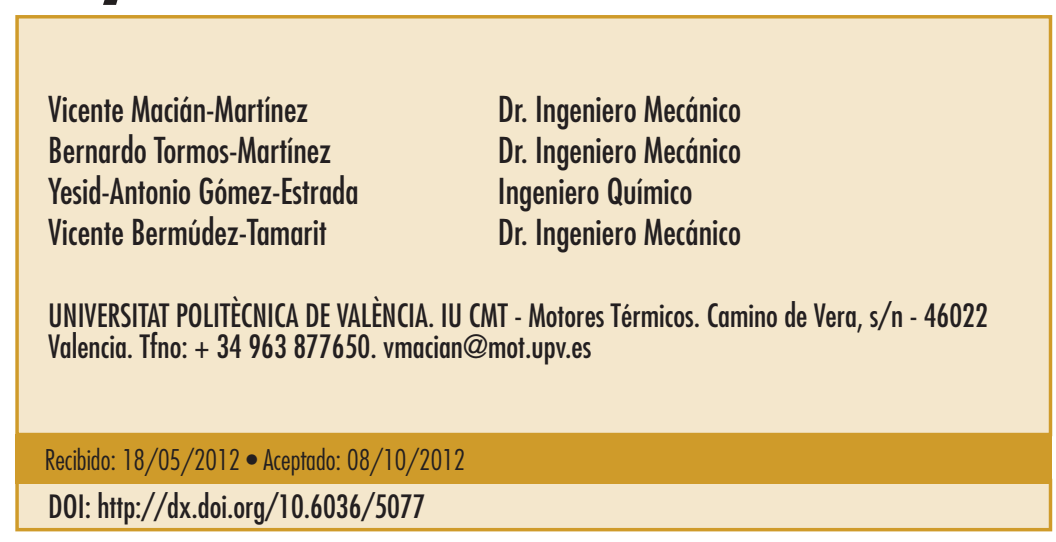

\section{A REVIEW OF DEGRADATION PROCESS ON COMPRESSED NATURAL GAS AND DIESEL ENGINES LUBRICANT OILS}

\section{ABSTRACT}

- In this study, a representative sample of urban buses, powered with CNG and diesel engine technologies and working on urban duty operation, have been studied in order to evaluate engine oil evolution of degradation. Main parameters evaluated have been related with oil degradation: oxidation, nitration, viscosity, Antioxidant additives depletion, antiwear, TAN, TBN and RUL Number. Results obtained have shown higher degradation rates for oils used on CNG engines than diesel engine. CNG engines studied showed a high thermal and mechanical stress, and lower oil sump volume. Experience with FT-IR technique on degradation study allowed to define optimal spectral ranges to quantify the changes of oxidation, nitration and fuel dilution problem in MCIA.

- Keywords: Degradation, compressed natural gas engine, oxidation, nitration, RUL - remaining useful life number and oil stress factor.

\section{RESUMEN}

En este estudio, una muestra representativa de autobuses de servicio urbano, con motorizaciones GNC y diesel, han sido estudiados con el fin de evaluar la evolución de la degradación de sus aceites. Los principales parámetros evaluados en relación con la degradación del aceite son: oxidación, nitración, viscosidad, agotamiento de los aditivos antioxidantes amínicos, antidesgaste, TAN, TBN y el número RUL.

Los resultados obtenidos evidencian mayores tasas de degradación de los aceites usados en los motores GNC frente a los motores diesel. Los motores de GNC estudiados presentan una mayor exigencia a sus aceites al contar con mayores solicitaciones térmicas, mecánicas, y menor volumen del cárter de aceite. La experiencia adquirida con la aplicación de la técnica de FTIR para el estudio de la degradación, ha permitido definir rangos espectrales óptimos para cuantificar los cambios de oxidación, nitración y presencia de combustible en MCIA.
Palabras clave: Degradación, motores de gas natural comprimido, oxidación, nitración, RUL - vida remanente del aceite, factor de estrés del aceite

\section{INTRODUCCIÓN}

Durante el desarrollo de este trabajo, dos tipos de motores de combustión interna alternativos han sido considerados: Gas Natural Comprimido (GNC) y diesel, como las configuraciones de mayor aplicación actual en flotas de transporte urbano. Estos motores presentan diferencias significativas como son: sus ciclos termodinámicos y las características de los combustibles empleados, pero sobre todo presentan algunas diferencias muy importantes de cara al proceso de degradación del aceite lubricante, a pesar de contar con el mismo periodo de servicio según sus fabricantes. Los motores GNC cuentan con una presencia muy baja de materia carbonosa en los productos de la combustión y carecen de problemas 
por dilución de combustible al utilizar un combustible gaseoso ${ }^{1}$. Con una combustión más "limpia" frente a la combustión de gasoil, los requerimientos para los aceites lubricantes en motores GNC cuentan con menores exigencias en cuanto a capacidad de detergencia y dispersancia, lo que permite a estos aceites ser formulados con menores niveles de contenido en cenizas, reduciendo la probabilidad de formación de depósitos que puedan provocar problemas de detonación. En contraposición, las mayores temperaturas de combustión en motores GNC, conducirán a mayores niveles de oxidación y nitración del aceite, como consecuencia de dichas solicitaciones térmicas llegando a ocasionar en muchos casos mayores niveles de degradación del aceite lubricante ${ }^{2,3}$.

A pesar de las ventajas presentadas en estos motores de tipo GNC, trabajos previos desarrollados por la línea de investigación del Departamento de Maquinas y Motores Térmicos de la UPV ${ }^{4-6}$ muestran que, al utilizar un aceite de tipo mineral en vehículos GNC con las características de los motores estudiados, hay una clara disminución de los paquetes de aditivos, incrementos en la acidificación del lubricante y elevados valores en la tasa de desgaste de algunos metales como el cobre y plomo, que podrían poner en riesgo el desempeño del motor. Frente a esta problemática, dos tipos de soluciones pueden ser adoptadas: reducción del periodo de servicio del aceite lubricante o la utilización de un aceite con una mejor formulación en cuanto a sus paquetes de aditivos y características de su base lubricante; permitiendo en ambos casos alcanzar los periodos de servicio establecidos por los fabricantes, sin que ello suponga un alto riesgo de fallo en los motores.

Este trabajo conducirá a la evaluación detallada de los procesos de degradación en estas dos tipologías de motores, utilizando para los motores GNC dos formulaciones de aceites lubricantes (Tipo I y II), y una formulación para los motores diesel (tipo III), que servirá como comportamiento de referencia. Todas las formulaciones de aceites lubricantes empleadas en el estudio son homologados por los fabricantes de los vehículos.

Teniendo en cuenta que las configuraciones de los motores involucrados son diferentes, se debe emplear algún parámetro que haga posible dicha comparación descartando los efectos de tamaño. Por una parte, se podría emplear la pre-

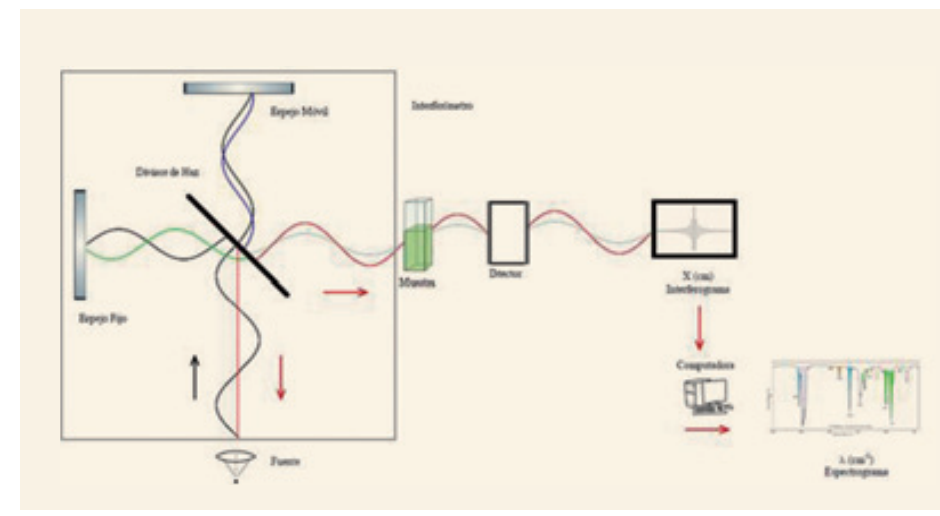

sión media efectiva (pme) o la potencia por unidad de área de pistón, de modo que pudiese representar una medida de la carga térmica o mecánica soportada por el motor, por otro lado podría utilizarse la potencia por unidad de cilindrada como un indicador de la efectividad relativa con la que se utiliza dicho volumen. Finalmente se optó por un parámetro que incluye los aspectos mencionados anteriormente más aquellos que están directamente relacionados con el aceite. Este parámetro es el llamado Factor de Stress del Aceite (OSF: Oil Stress Factor). Este parámetro ha sido utilizado como estimador del potencial estrés que sufre el aceite y permite cierta correlación con su posible degradación como consecuencia del diseño del motor ${ }^{4,7,8}$. El OSF considera la relación existente entre la potencia específica por cilindrada, el periodo de servicio de aceite y el volumen de cárter, como se muestra en la ecuación siguiente.

$$
\operatorname{OSF}\left(\frac{k W k m}{l^{2}}\right)=\frac{\text { Potencia }[k W]}{\text { Cilindrada }[l]} \times \frac{\text { Periodo de Servicio }[\mathrm{km}]}{\text { Volumen del Cárter }[l]}
$$

\section{EQUIPOS Y METODOLOGIAS}

Diferentes técnicas de análisis de aceite han sido utilizadas durante el estudio de la degradación de aceites lubricantes en motores de combustión interna de tipo GNC y diesel. Una de las principales y en las cuales se ha centrado parte de estos estudios es la espectrometría infrarroja por transformada rápida de Fourier (FT-IR).

FT-IR es la principal técnica analítica para determinar productos de la degradación (oxidación, nitración), así como el agotamiento de los paquetes aditivos en los aceites lubricantes que han sido utilizados. El resto de técnicas utilizadas (voltamperometría, potenciometría, entre otras) cuentan con mayor tiempo de aplicación en este campo y se ha profundizado mucho mas a nivel de normativas y estandarización, permitiendo comparar los resultados que se desarrollan entre laboratorios, algo que es mucho más complicado en la actualidad con los resultados que se obtienen con los ensayos a través de FT-IR. A continuación se presentan las principales características de los equipos, así como las técnicas analíticas que han sido utilizadas en cada uno de ellos.

Fig. 1: Diagrama de un espectrómetro por transformada de Fourier

Un espectrómetro por transformada de Fourier consta de tres elementos básicos: una fuente luminosa, un interferómetro de $\mathrm{Mi}$ chelson y un detector. La radiación infrarroja que se genera en la fuente luminosa, incide sobre un divisor de haz. El haz incidente se divide en dos haces perpendiculares de igual energía, uno de los cuales incide sobre el espejo móvil y el otro sobre el espejo fijo. El haz resultante pasa a través de la muestra, en donde sucede una absorción selectiva de longitudes de onda llegando al detector. La fase final del proceso del espectrograma se completa realizando un espectro del ruido de fondo (background) mediante la recogida de un interferograma, siguiendo un procesamiento de los datos mediante transformada de Fourier para obtener finalmente el espectrograma de la muestra. 


\subsection{ESPECIFICACIONES DE LOS EQUIPOS, TÉCNICAS ANALÍTICAS Y METODOLOGÍAS}

\subsubsection{Espectrometría infrarroja por transformada rápi- da de Fourier (FT-IR)}

Las medidas de los niveles de oxidación, nitración y paquetes de aditivos de tipo amínicos y antidesgaste fueron realizadas a través de un equipo de espectrometría infrarroja por transformada rápida de Fourier, cuyas características son presentadas en la Tabla 1.

\begin{tabular}{lc}
\hline \multicolumn{1}{c}{ Características } & FT-IR iPAL \\
\hline Rango Espectral & $4.700 \mathrm{~cm}^{-1}$ a $590 \mathrm{~cm}^{-1}$ \\
\hline Resolución & $4 \mathrm{~cm}^{-1}$ \\
\hline Escaneo de la muestra & 128 \\
\hline Escaneo del ambiente & 128 \\
\hline Longitud del paso de luz $\mathrm{mm}(\mu \mathrm{m})$ & $0,1(100)$ \\
\hline Material de la celda & Seleniuro de Zinc $(\mathrm{ZnSe})$ \\
\hline Interface & Transmisión - TumbllR \\
\hline Apodización & Triangular \\
\hline
\end{tabular}

Tabla 1: Características principales del FT-IR - iPAL A2 Technologies se línea base, las mediciones de la altura y área del pico se realizarán sobre el eje de abscisas.

En todos los casos se calcularon los niveles de los parámetros de oxidación, nitración y paquetes de aditivos mediante la utilización de una línea base doble y los resultados fueron reportados en términos de absorbancia.

Con el fin de estudiar la degradación de los aceites lubricantes se deben identificar aquellos subproductos que presentan, debido al grupo $\mathrm{C}=\mathrm{O}$, rangos de absorción en un intervalo de frecuencias entre $1.850 \mathrm{~cm}^{-1}$ y $1.620 \mathrm{~cm}^{-1}$, ya que conforme aumenta la degradación de estos aceites lubricantes va aumentando la concentración de estos productos oxigenados y por consiguiente provoca un aumento de la absorbancia en esta zona ${ }^{9-12}$.

Con el fin de identificar y establecer los límites de detección por FT-IR (rangos espectrales) para los subproductos que provienen de la degradación térmica, se procedió a degradar bajo condiciones controladas en el laboratorio una serie de muestras de aceite lubricante, de tal manera que se pudo observar los cambios y las tendencias de los espectros. Este proceso de degradación térmica en el laboratorio fue llevado a cabo en baño térmico con un controlador de temperatura, en el cual se introdujo un beaker con un volumen de muestra de $100 \mathrm{ml}$. Se utilizó un termómetro ASTM 3C en el interior del beaker para llevar un mejor control de la temperatura. Los ensayos se realizaron utilizando dos mé-

\begin{tabular}{c|c|c|c|c}
\hline & Oxidación & Nitración & $\begin{array}{c}\text { Antioxidantes } \\
\text { Amínicos (AA) }\end{array}$ & Antidesgaste \\
\hline Metodología & ASTM D7214 & ASTM D 2412 & CMT-0124.12 $\left(^{*}\right)$ & CMT-0120.12 $\left(^{*}\right)$ \\
\hline Unidades & $\left(\right.$ Abs $\left.\mathrm{cm}^{-1}\right) / 0.1 \mathrm{~mm}$ & $\left(\right.$ Abs $\left.\cdot \mathrm{cm}^{-1}\right) / 0.1 \mathrm{~mm}$ & $\left(\right.$ Abs $\left.\cdot \mathrm{cm}^{-1}\right) / 0.1 \mathrm{~mm}$ & $\left(\right.$ Abs $\left.\cdot \mathrm{cm}^{-1}\right) / 0.1 \mathrm{~mm}$ \\
\hline Rango de frecuencia cm ${ }^{-1}$ & $1.725-1.650$ & $1.650-1,600$ & $1.550-1,490$ & $1.026-941$ \\
\hline Línea base $1 \mathrm{~cm}^{-1}$ (Inicio - Fin) & $2.200-1.900$ & $2.200-1.900$ & $2.200-1.900$ & $1.100-1.098$ \\
\hline Línea base $2 \mathrm{~cm}^{-1}$ (Inicio - Fin) & $650-615$ & $650-615$ & $650-615$ & $911-909$ \\
\hline
\end{tabular}

Tabla 2: Metodologías aplicadas en los ensayos de FT-IR

(*) Designación correspondiente a metodologías internas del CMT. CMT-0124.12 y CMT-0120.12 están basadas en la normativa ASTM E 2412

Las metodologías aplicadas a través de esta técnica para cada una de las variables medidas se detallan en la Tabla 2.

La cuantificación de los diferentes parámetros a través de FT-IR se ha realizado a través de la medida de la altura $(\mathrm{PH}$ - Peak Heigh) y del área de pico (PA - Peak Area), utilizando una línea de referencia.

Esta línea de referencia puede ser: una línea base única, la cual constituye una línea paralela al eje de abscisas que se inicia en el punto más bajo del espectro dentro de un intervalo definido. Los intervalos de la línea base se definen en un rango ligeramente mayor $\left(+/-10 \mathrm{~cm}^{-1}\right)$ al elegido para el cálculo de la variable (oxidación, nitración, etc.). El otro caso es utilizar una línea base doble, la cual se traza entre los intervalos donde se definen estas líneas base, seleccionando de cada intervalo el punto más bajo. En el caso de no tomar- todos de calentamiento: uno isotérmico y uno dinámico con los siguientes valores de temperatura $200{ }^{\circ} \mathrm{C}, 220{ }^{\circ} \mathrm{C}, 240$ ${ }^{\circ} \mathrm{C}$ y $260{ }^{\circ} \mathrm{C}$. Al calentar la muestra se consigue degradar el aceite de tal manera que se incrementan sus niveles de oxidación y sus paquetes de aditivos antioxidantes se agotan. El tiempo total de degradación fue de 24 horas, durante el cual se muestreaba con una frecuencia de 3 horas. Posteriormente, dichas muestras eran analizadas a través de FT-IR, TAN, TBN y medida de la viscosidad cinemática. Todo el proceso fue realizado en el interior de una cabina cerrada con el fin de evitar la suspensión de partículas así como una posible contaminación de las muestras.

Como se observa en la Fig. 2, estos productos provenientes de una degradación isotérmica a $260{ }^{\circ} \mathrm{C}$, presentan cambio significativos en el rango entre $1.725 \mathrm{~cm}^{-1}$ y $1.650 \mathrm{~cm}^{-1}$, 
motivo por el cual será este rango el utilizado para la medida de los niveles de oxidación presentes en los aceites.

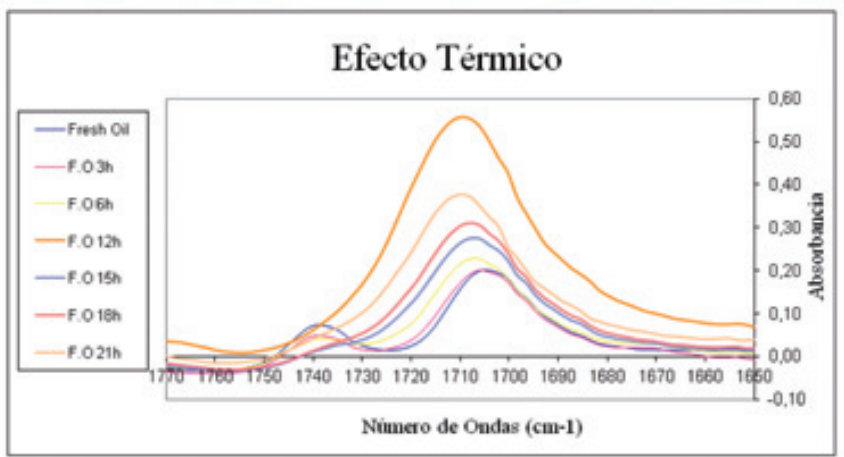

Fig. 2: Efecto de la temperatura en la zona de oxidación

Además de producirse cambios en esta zona, se obtienen otros productos que provienen de las reacciones con el nitrógeno presente en el aire, ocasionando la nitración del aceite. Esta reacción tiende a incrementarse cuando las temperaturas son más altas. En condiciones reales de operación se suelen asociar con el incremento del "blow-by", pues los gases calientes de combustión reaccionan con el aceite ó cuando se tienen dosados pobres. La medida de la nitración con FT-IR se ha realizado en la zona del espectro entre $1.650 \mathrm{~cm}^{-1} \mathrm{y}$ $1.600 \mathrm{~cm}^{-1}$

La otra variable que produce cambios significativos en esta zona del rango espectral $\left(1.850 \mathrm{~cm}^{-1}-1.620 \mathrm{~cm}^{-1}\right)$, es la dilución por combustible en motores diesel, cuando se está utilizando biocombustibles. A pesar de que los combustibles fósiles no presenten dentro de sus estructuras compuestos que puedan producir cambio en esta zona espectral, la incorporación de los biodiesel hoy en día (hasta un 7 \% $/ \mathrm{v}$, según normativa Europea - UNE - EN 590) ${ }^{13}$, hace que estos lleguen a presentar alteraciones como consecuencia de la presencia de los esteres como se puede observar en la Fig. 3. En esta ocasión se ha procedido a contaminar una muestra del aceite lubricante tipo III con distintas concentraciones de biodiesel. Por consiguiente, viendo los efectos que producen los potenciales problemas de dilución en aceites lubricanes para motores diesel, se utilizará la zona entre $1.770 \mathrm{~cm}^{-1} \mathrm{y}$ $1.725 \mathrm{~cm}^{-1}$ para cuantificar los dichos problemas en lugar de

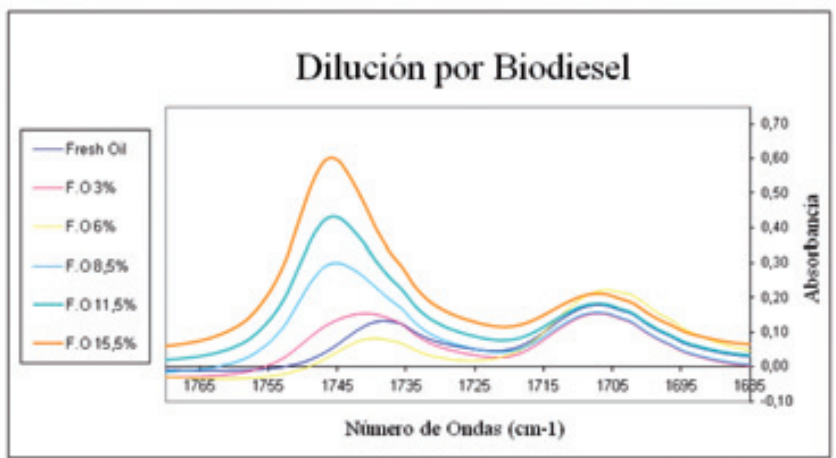

Fig. 3: Nuevos rangos para la detección de la dilución por biodiesel otras zonas del espectro $\left(815 \mathrm{~cm}^{-1}-745 \mathrm{~cm}^{-1}\right.$, como se puede observar en la Fig. 4) que suelen utilizase para combustibles convencionales según normativas.

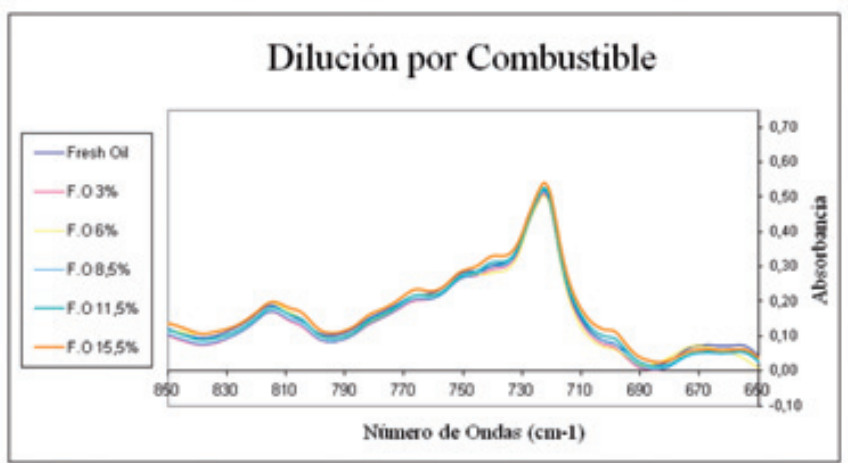

Fig.4: Rangos convencionales para la detección de la dilución por combustible

\subsubsection{Viscosidad}

La viscosidad es una de las características más importantes de los aceites lubricantes y se puede definir como la oposición de un líquido a fluir debido al rozamiento interno de sus propias moléculas. Para calcular la viscosidad cinemática se recurre al uso de viscosímetros siguiendo la metodología ASTM D445. Todos los ensayos utilizaron viscosímetros para líquidos opacos y fueron realizados a una temperatura de $40^{\circ} \mathrm{C}$.

\subsubsection{TAN y TBN}

Los niveles de acidez (Total Acid Number - TAN) y de alcalinidad (Total Basic Number - TBN) han sido obtenidos mediante un equipo automático de titulación potenciométrica ORION 950, utilizando una metodología de "primera derivada". Las soluciones de titulación utilizadas en este ensayo corresponden a las establecidas por las normativas para los cálculos de acidez (ASTM D 664) y alcalinidad (ASTM D 2896).

\subsubsection{Numero de RUL (Remaining Useful Life)}

El numero de RUL o vida remanente del aceite lubricante ha sido calculado a partir de una técnica voltamperométrica. Para la medida del numero RUL, se utilizó un equipo de FLUITEC - RULER $\AA$, el cual se basa en la técnica de la voltamperometría lineal de barrido. Esta técnica instrumental analítica permite oxidar o reducir compuestos disueltos en el seno de un disolvente mediante la aplicación de un potencial positivo o negativo, respectivamente. En este método voltamperométrico se varía el potencial aplicado con el tiempo, y a esta variación se le llama velocidad de barrido pudiendo variar desde $\mathrm{mV} / \mathrm{s}$ hasta $1.000 .000 \mathrm{~V} / \mathrm{s}$.

Una de las aplicaciones de esta técnica es determinar la concentración de aditivos antioxidantes (AO) en el seno de un lubricante aplicando un potencial positivo entre unos rangos de potencial $(0,0-1,7 \mathrm{~V})$ y velocidades de $0,1 \mathrm{~V} / \mathrm{s}$ para oxidarlos. La oxidación de los AO se producirá en función de su potencial de oxidación $\left(\mathrm{E}_{\mathrm{ox}}\right)$, definido como la facilidad 
que poseen para desprenderse de un electrón. Los resultados se expresan a través de un gráfico llamado voltamperograma (Fig.5).

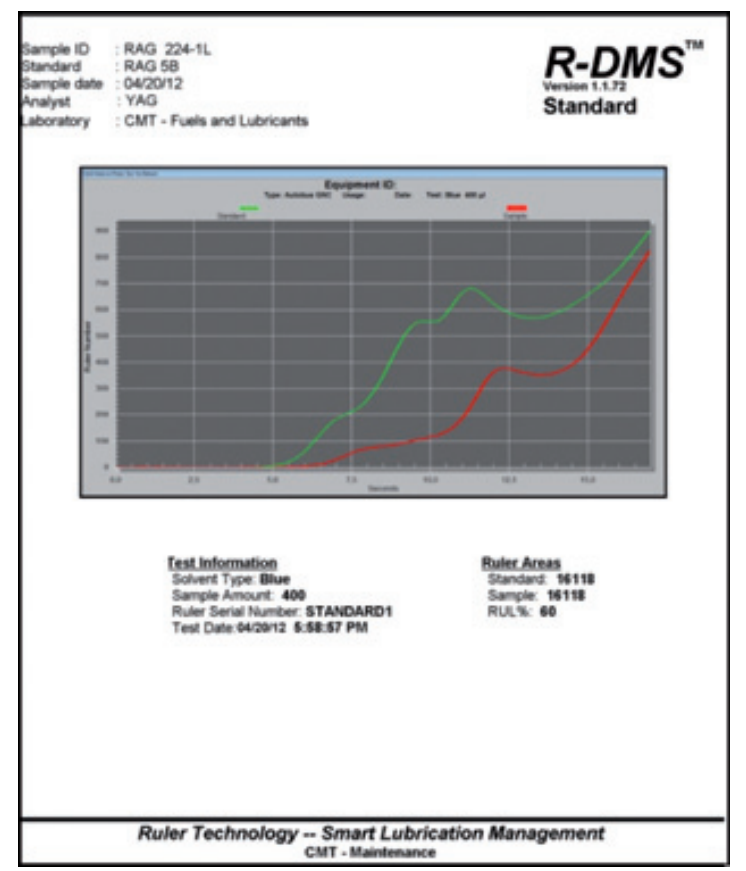

Figura 5: Voltamperograma de un aceite lubricante

El número RUL se reporta como la diferencia en términos de \% del área del voltamperograma de la muestra de referencia y la analizada.

En el caso de los MCIA, los aditivos antioxidantes que suelen identificarse por medio de esta técnica corresponde a los grupos amínicos y ZDDP, los cuales presentan unos tiempo de oxidación entre $7-9$ segundos para los aditivos de tipo amínicos y de 11 - 12 segundos los ZDDP (Figura 6). Los aditivos antioxidantes de tipo fenólicos son muy difíciles de detectar y además suelen confundirse con el ruido de fondo de la corriente ${ }^{14-17}$.

\subsection{CARACTERÍSTICAS DE LOS VEHÍCULOS Y ACEITES LUBRICANTES}

Todas las muestras analizadas proceden de 15 vehículos que operan en una flota de transporte urbano con motores

\begin{tabular}{|c|c}
\hline & Antioxidantes (Número de RUL) (A0) \\
\hline Metodología & CMT-0091.11 $\left(^{*}\right)$ \\
\hline Técnica analítica & Voltamperometría \\
\hline Unidades & $\%$ \\
\hline
\end{tabular}

Tabla 3: Metodologías aplicadas para los ensayos de número de RUL

*) Designación correspondiente a metodologías internas del CMT. CMT-0091.11 está basada en la normativa ASTM D 6810 Standard Test Method for Measurement of Hindered Phenolic Antioxidant Content in Non-Zinc Turbine Oils by Linear Sweep voltammetry, y la normativa ASTM D 6971 Standard Test Method for Measurement of Hindered phenolic and Aromatic Amine Antioxidant Content in Non-zinc Turbine Oils by Linear Sweep voltammetry

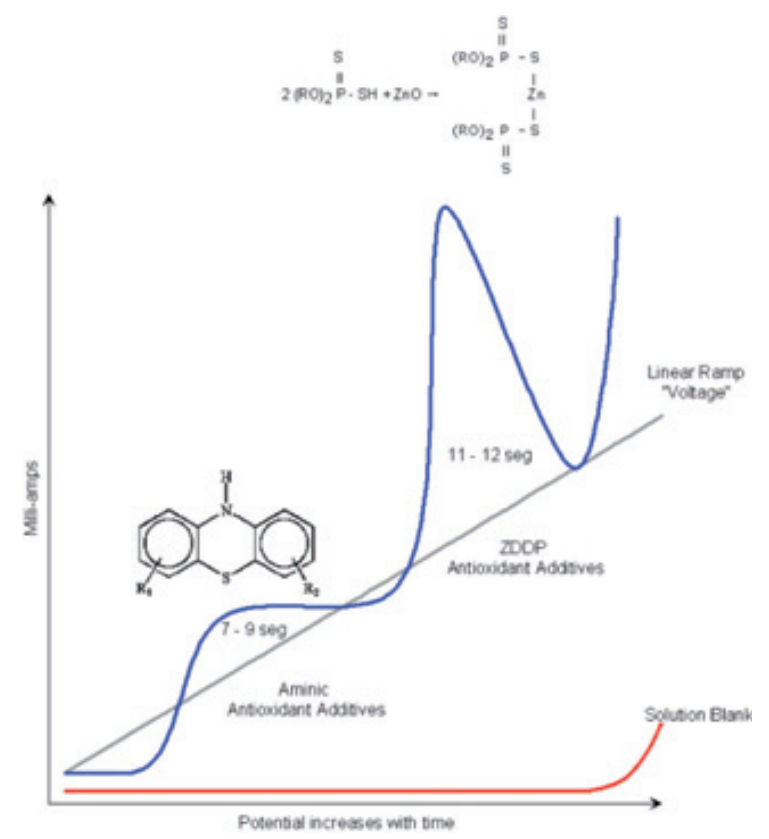

Fig. 6: Aditivos antioxidantes típicos en aceites de motor

GNC y diesel, con el fin contrastar los resultados obtenidos de una tecnología frente a otra. Este tipo de motores soportan lo que se denomina un tipo de servicio severo, debido a sus constantes paradas y arranques, largos periodos de trabajo al ralentí y una velocidad media comercial de $12,7 \mathrm{~km} / \mathrm{h}$. Los vehículos son operados en dos turnos de 8 horas. La ciudad donde se han llevado a cabo las pruebas es totalmente llana y no se pueden asociar diferencias sustanciales asociadas a la ruta efectuada.

Con el fin de contar con un mejor análisis de los resultados obtenidos, todos los vehículos mantuvieron las mismas condiciones de operación (rutas de servicio) durante todo el tiempo del estudio.

Las características principales de los motores de los vehículos y los aceites lubricantes son presentadas en la Tabla 4 y 5 .

\begin{tabular}{lcc}
\hline \multicolumn{1}{c}{ Características } & Motores GNC & Motores Diesel \\
\hline Tipo & $\begin{array}{c}\text { Inyección Indirecta } \\
\text { / Turboalimentado }\end{array}$ & $\begin{array}{c}\text { Inyección Directa } \\
\text { / Turboalimentado }\end{array}$ \\
\hline Numero de cilindros & 6 & 6 \\
\hline Diámetro / Carrera (mm) & $115 / 125$ & $128 / 155$ \\
\hline Cilindrada (cc) & 7.790 & 11.967 \\
\hline Potencia CV & $270 @ 2.000$ & $220 @ 1.900$ \\
& $\mathrm{rpm}$ & $\mathrm{rpm}$ \\
\hline pme (bar) & 15,4 & 11,6 \\
\hline Periodo de servicio (km) & 30.000 & 30.000 \\
\hline Volumen del carter (I) & 23 & 31 \\
\hline OSF (kWkm/12) & 33.488 & 20.756 \\
\hline Tipo de aceite lubricante & $\mathrm{I} \mathrm{y} \mathrm{II}$ & $\mathrm{III}$ \\
\hline
\end{tabular}

Tabla 4: Características principales de los motores 
Todos los vehículos utilizaron aceites de tipo mineral cuyas características de son presentadas en la Tabla 5.

Todas las muestras (250) se tomaron con recipiente nuevos de $125 \mathrm{ml}$ con un periodo de $5.000 \mathrm{~km}$, además de tomar muestras al final del periodo de servicio. Las muestras fueron extraídas desde el cárter por medio de una bomba manual "Vampire", que contaba con un recipiente previamente etiquetado y un tubo nuevo flexible de poliuretano (PUR) para cada una de las muestras. Los recipientes se rellenaron con aproximadamente $100 \mathrm{ml}$ de muestra, de manera que posteriormente pudiera realizarse una agitación intensa antes de realizar cualquier tipo de medida. Las muestras que fueron tomadas al finalizar el periodo de servicio, eran tomadas por personal técnico de la empresa de transporte, que rellenaban un recipiente directamente con el aceite que se drena, tras dejar fluir al menos tres segundos el aceite antes de tomar la muestra.

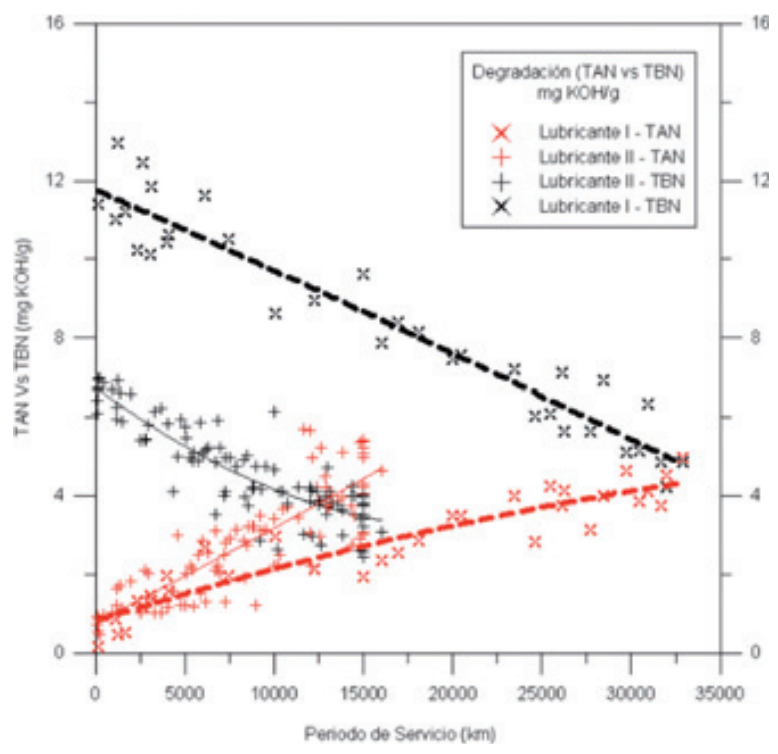

Fig. 7: Comparativa TAN / TBN en motores GNC

\section{RESULTADOS}

A continuación se presentan los resultados obtenidos en campo, en función de las variables analizadas.

\subsection{RESULTADOS DE LOS NIVELES DE TAN / TBN}

Las Figs. 7 y 8, muestran la evolución de las medidas de acidez y del agotamiento de la reserva alcalina en $\mathrm{mg} \mathrm{KOH} / \mathrm{g}$ de las muestras analizadas para los motores GNC y diesel respectivamente.

Podemos inferir en primera instancia que la intersección de las curvas de tendencia de las medidas de TAN y TBN, muestran los periodos de servicio para cada uno de los aceites lubricantes. Como podemos observar el aceite tipo II, muestra una clara reducción del periodo establecido hasta un $50 \%$ aproximadamente $(15.000 \mathrm{~km})$. Alcanzar los periodos de servicio establecidos por el fabricante $(30.000 \mathrm{~km})$ utilizando esta formulación podría suponer graves riesgos de

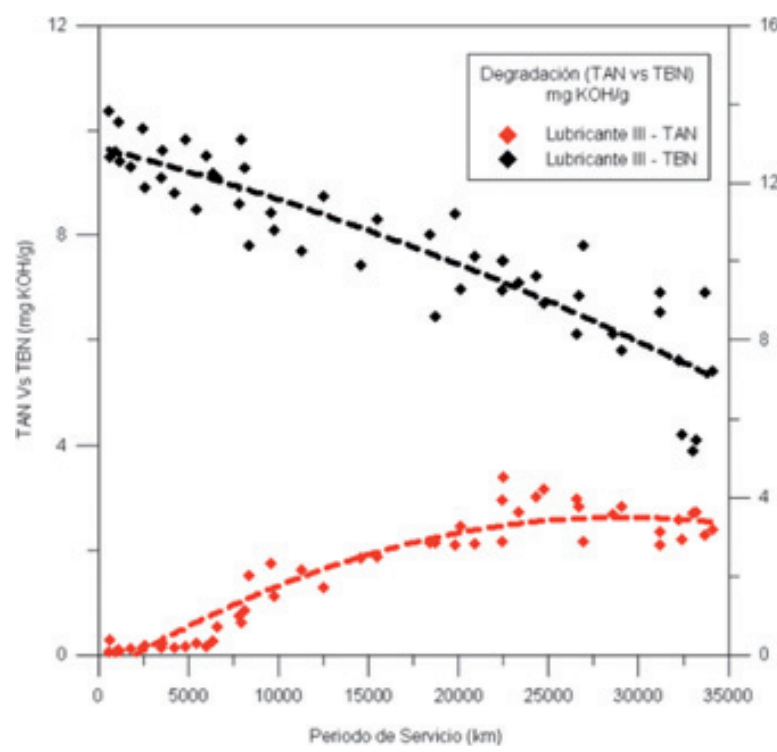

Fig. 8: Comparativa TAN / TBN en motores Diesel

\begin{tabular}{|c|c|c|c|}
\hline Características & Tipo I & Tipo II & Tipo III \\
\hline Grado SAE & $10 W 40$ & $15 \mathrm{~W} / 40$ & $15 \mathrm{~W} / 40$ \\
\hline Densidad a $15^{\circ} \mathrm{C}\left(\mathrm{kg} / \mathrm{m}^{3}\right)$ & 865 & 885 & 881 \\
\hline Viscosidad a $40^{\circ} \mathrm{C}$ (cSt) & 91,8 & 112,0 & 108,0 \\
\hline Viscosidad a $100^{\circ} \mathrm{C}$ (cSt) & 14,3 & 14,5 & 14,5 \\
\hline Índice de Viscosidad & 160 & $125 \mathrm{~min}$. & $130 \mathrm{~min}$. \\
\hline T.B.N. (mg KOH/g) & 13,2 & 7,0 & 10 \\
\hline Aditivos amínicos (Abs $\left.\mathrm{cm}^{-1} / 0.1 \mathrm{~mm}\right)$ & $17.991^{*}$ & $12.978^{*}$ & $1.275^{*}$ \\
\hline Antidesgaste $($ Abs cm-1 $/ 0.1 \mathrm{~mm})$ & $8.048^{*}$ & $10.903^{*}$ & $12.950^{*}$ \\
\hline Punto de inflamación, vaso abierto $\left({ }^{\circ} \mathrm{C}\right)$ & $>220$ & 215 & $215 \min$. \\
\hline Pour point $\left({ }^{\circ} \mathrm{C}\right)$ & $<-33$ & -27 & $-27 \max$ \\
\hline Especificaciones & IVECO 18-1809 & API CF-4 & ACEA E7/E5, API Cl-4/CH-4/SL \\
\hline
\end{tabular}

Tabla 5: Características principales de los aceites lubricantes

(*) Estos resultados corresponden a medidas realizadas en el laboratorio por medio de la técnica de FT-IR 


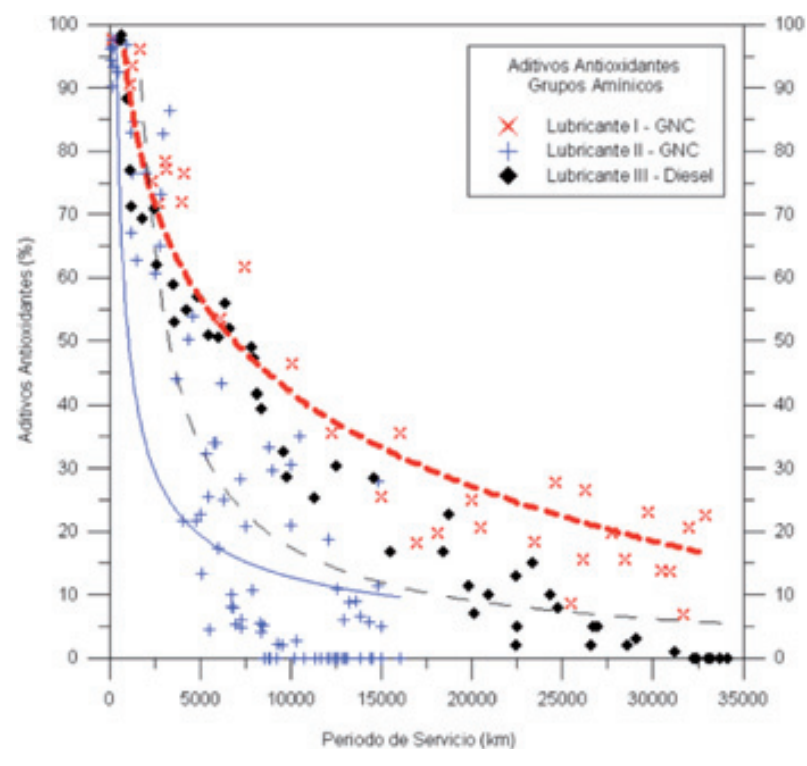

Fig.9: Evolución de los aditivos de tipo amínicos por FT-IR

fallo en el motor. Se puede atribuir el mejor comportamiento que presenta la formulación I frente al tipo II, a sus paquetes de aditivos y muy seguramente a su base lubricante.

Cabe destacar que la formulación I cuenta con niveles superiores de TBN que el tipo II, permitiendo de esta manera neutralizar los elevados niveles de acidificación del aceite al final del periodo de servicio. Los motores diesel, muestran sin lugar a dudas, una menor exigencia desde el punto de vista de la acidez del aceite, viéndose muy poco afectados por esta variable. Su reserva alcalina alcanza sin ningún tipo de problema los periodos que se ha establecido para estos motores pudiendo alcanzar niveles superiores si hiciéramos una extrapolación de los datos obtenidos.

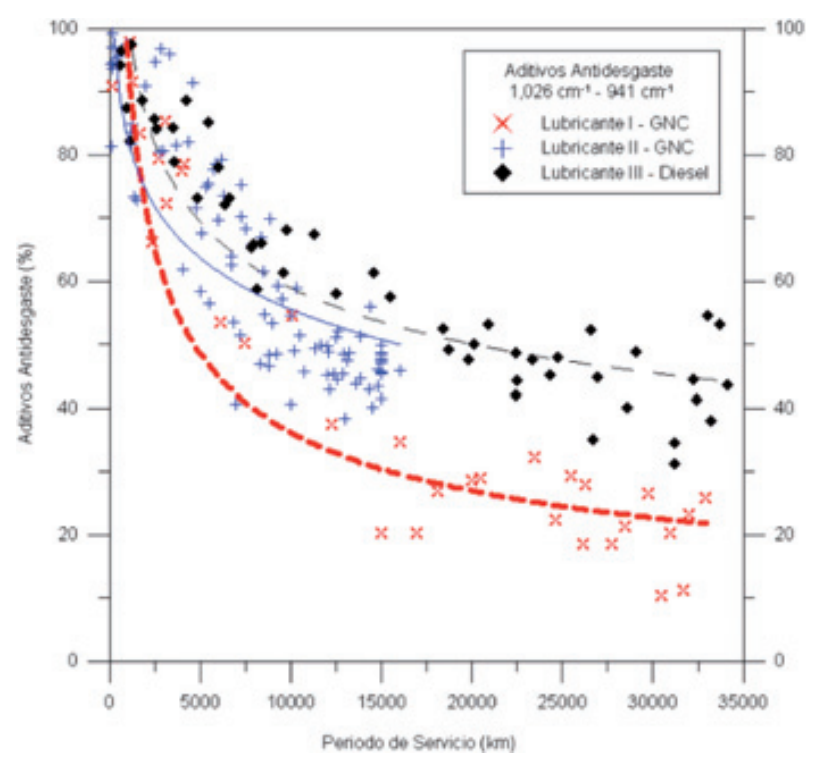

Fig. 10: Evolución de los aditivos antidesgaste por FT-IR

\subsection{RESULTADOS DE LOS PAQUETES DE ADITIVOS POR FT-IR Y VOLTAMPEROMETRÍA}

Las Figs. 9 y 10, presentan el comportamiento de los paquetes aditivos antioxidantes amínicos y antidesgaste para las dos tipologías de motores, a través de la técnica de FTIR. La Figura 9 muestra una marcada diferencia entre las tasas de agotamiento en los aceites lubricantes tipo I y II, lo que evidencia una mayor resistencia de la formulación tipo I frente a las solicitaciones dadas por el motor, además de contar con mayores concentraciones en sus paquetes de aditivos antioxidante de tipo amínico como se puede observar en la Tabla 5. Los aditivos antidesgaste, representados en la Fig. 10, muestran un mayor consumo en los motores

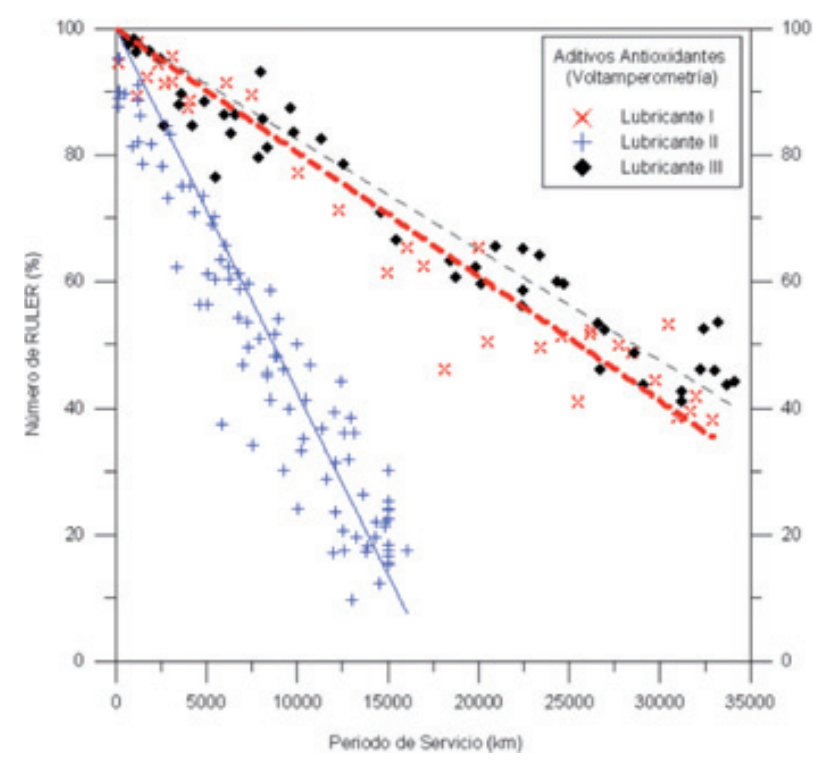

Fig. 11: Evolución de los aditivos antioxidantes por Voltamperometría

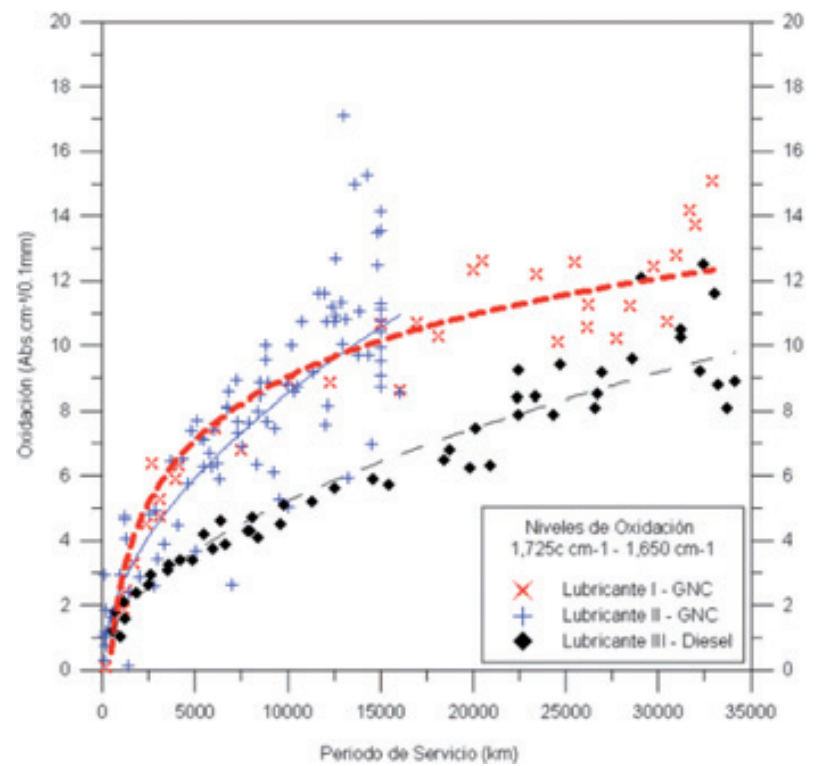

Fig. 12: Evolución de los niveles de oxidación mediante FT-IR 
GNC, como una consecuencia del posible desgaste que puedan estar sufriendo estas configuraciones de motor, así como contar con menores concentraciones de este tipo de aditivos en su formulación. Los motores diesel siguen presentando al igual que los consumos de la reserva alcalina un mejor comportamiento frente a las condiciones de operación del motor.

Las medidas de los paquetes de aditivos antioxidantes por medio de la técnica de voltamperometría lineal de barrido, que involucra no solo aquellos aditivos de tipo amínico, sino también los de tipo ZDDP como se ha expuesto previamente, siguen manteniendo un comportamiento similar al observado en las medidas por FT-IR, como podemos observar en la Fig. 11.

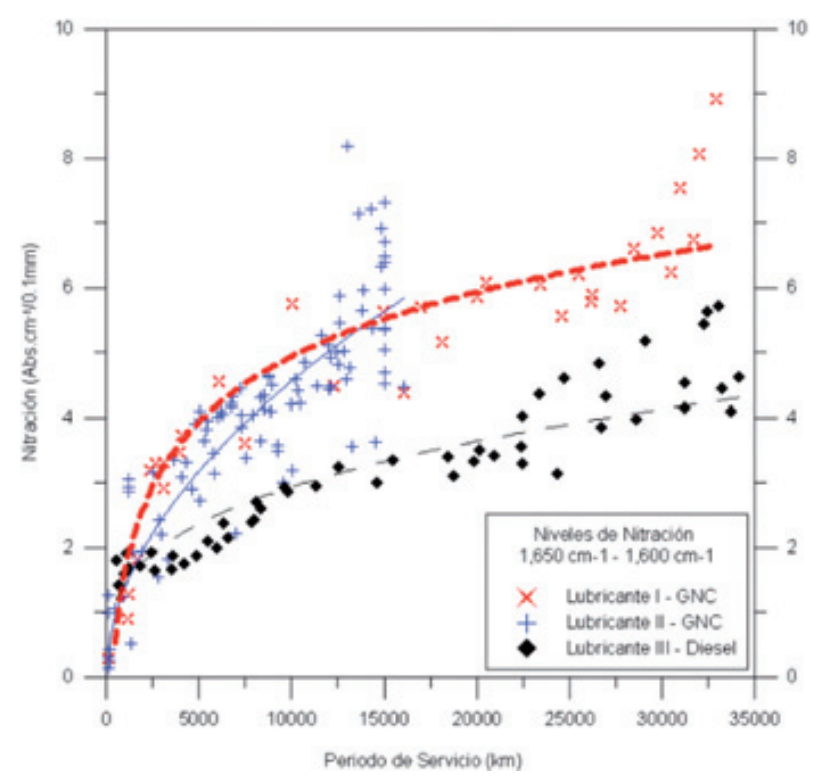

Fig. 13: Evolución de los niveles de nitración mediante FT-IR

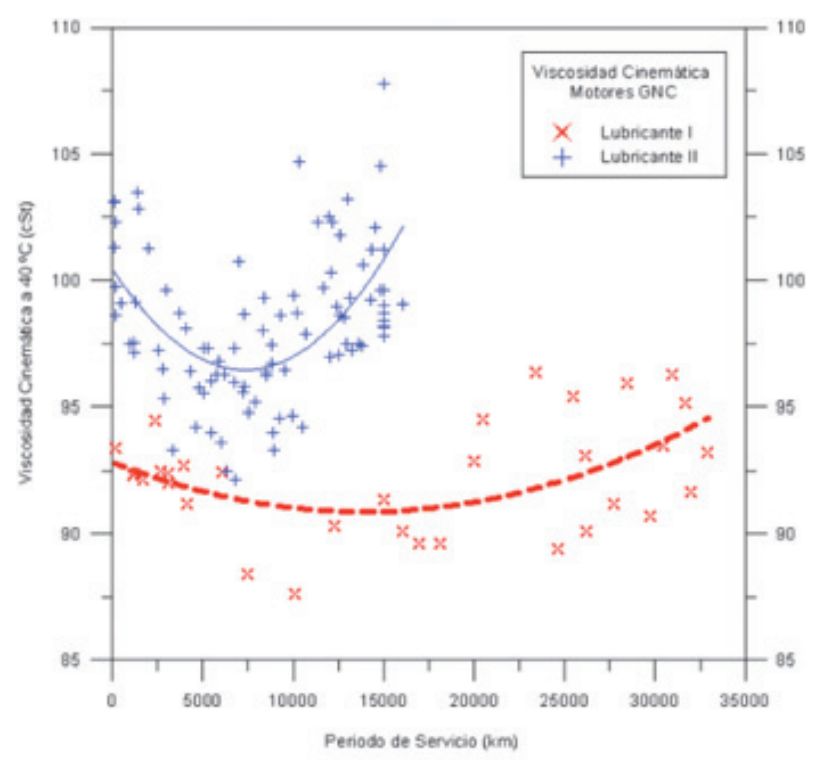

Fig. 14: Evolución de la viscosidad cinemática en motores GNC

\subsection{RESULTADOS DE LOS NIVELES DE OXIDACIÓN Y NITRACIÓN POR FT-IT}

Como se puede observar en las Fig.s 12 y 13, los resultados obtenidos para los niveles de oxidación y nitración, tomando como punto comparativo $15.000 \mathrm{~km}$, presenta mayores niveles de degradación en los motores de GNC frente a los motores diesel, como una consecuencia directa de la elevada solicitación a la que están sometidos los aceites lubricantes en esta configuración de motores y sobre todo al agotamiento que presentan sus paquetes de aditivos. Se llegan a obtener valores similares de oxidación y nitración para el lubricante II respecto al I, cuando su periodo de servicio se encuentra a un $50 \%(15.000 \mathrm{~km})$ del recomendado por el fabricante. En todos los casos, los niveles de oxidación y nitración mantienen una correlación lineal en función del agotamiento de los paquetes de aditivos antioxidantes como se ha podido comprobar en otros estudios ${ }^{4}$.

\subsection{RESULTADOS DE VISCOSIDAD CINEMÁTICA}

Los resultados para la viscosidad cinemática a $40{ }^{\circ} \mathrm{C}$ se presentan en la Figura 14 y 15 para los motores GNC y diesel respectivamente. La evolución de las muestras de los motores de GNC se puede dividir en dos partes: inicialmente hay una reducción en el valor de la viscosidad, relacionado con el efecto de cizallamiento de los aditivos mejoradores del índice de viscosidad, sin embargo, después de este período inicial hay una tendencia creciente en las mediciones de viscosidad, hasta tal punto de obtener valores en el período de cambio muy similares a los del aceite nuevo como consecuencia del proceso de oxidación del aceite (especies con un alto peso molecular) que es la principal causa del espesamiento del aceite ${ }^{18}$. En el caso de los motores diesel, presentan una evolución típica de esta variable en función del periodo de servicio en aceites multigrado, donde se observa una clara reducción en los valores de la viscosidad como consecuencia de los efectos del cizallamiento ${ }^{5,19}$.

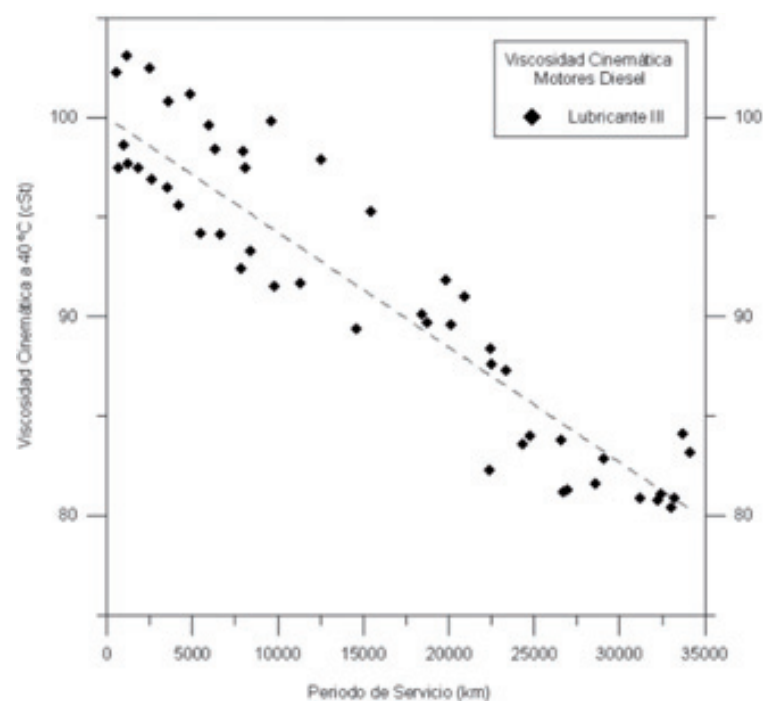

Fig. 15: Evolución de la viscosidad cinemática en motores diesel 
Tras haber analizado el comportamiento de la degradación mediante distintas técnicas analíticas en motores GNC y diesel, se procedió a realizar un pequeño análisis de los resultados de la cuantificación del desgaste del motor a través de la técnica de espectroscopía de emisión óptica de plasma acoplado inductivamente (ICP-OES), como se puede observar en la Fig. 16. Estos análisis fueron llevados a cabo en un laboratorio externo que presta dicho servicio al operador de los vehículos. En esta figura se representaron los niveles de desgaste de aquellos metales que presentaron mayores concentraciones e incidencias dentro del proceso de degradación del aceite en motores GNC que utilizaban del aceite tipo II. El cobre, hierro y plomo además de contar con concentraciones bastante apreciables, tienen la facilidad de interactuar como catalizadores durante el proceso de degradación oxidativa de los aceites lubricantes en motores de combustión interna, siendo el cobre sin lugar a duda unos de los catalizadores más influyentes dentro del proceso de degradación oxidativa $^{6,18}$. Los valores típicos que podrían llegar a obtenerse en condiciones extremas como las presentadas en este estudio estarán alrededor de las 80 ppm de $\mathrm{Cu}$.

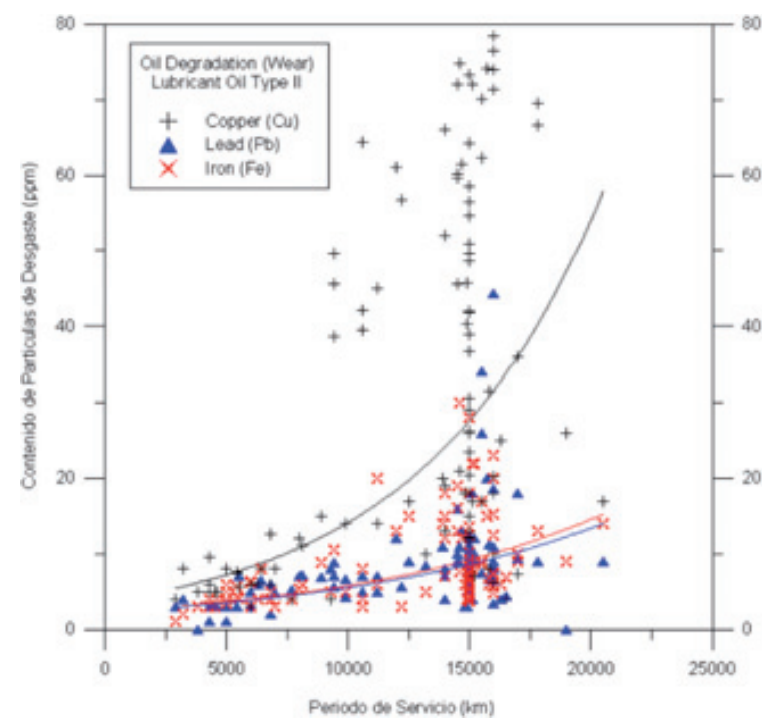

Figura 16: Evolución de los metales de desgaste en motores GNC por medio de las técnica ICP-OES

\section{CONCLUSIONES}

Los motores GNC muestran mayores exigencias térmicas que los motores diesel, y por consiguiente, requieren de formulaciones especificas de sus aceites lubricantes, para cumplir con los periodos de servicio recomendados por los fabricantes. Utilizar formulaciones que presenten bajas configuraciones en sus paquetes de aditivos, como la formulación tipo II, puede impedir alcanzar los periodos de servicio establecidos por los fabricantes, ocasionando mayores operaciones de mantenimiento y por consiguiente mayores costes.
El análisis de los metales de desgaste, y en especial del $\mathrm{Cu}, \mathrm{Pb}$ y $\mathrm{Fe}$, deben ser considerados en el diagnostico de los motores de GNC y diesel como uno de los parámetros principales a controlar dentro de la técnica de ICP-OES. Los altos niveles en los metales de desgaste del aceite tipo II y en especial de $\mathrm{Cu}$ que están en las muestras tomadas al final del periodo de servicio, obedecen en gran medida al agotamiento que ha presentado en sus paquetes de aditivos antioxidantes y antidesgaste, así como su correspondiente acidificación. Los aditivos antioxidantes de tipo amínico tienen entre otras funciones la de reducir la corrosión de metales al interactuar con otros aditivos en esta etapa final de su tiempo de uso.

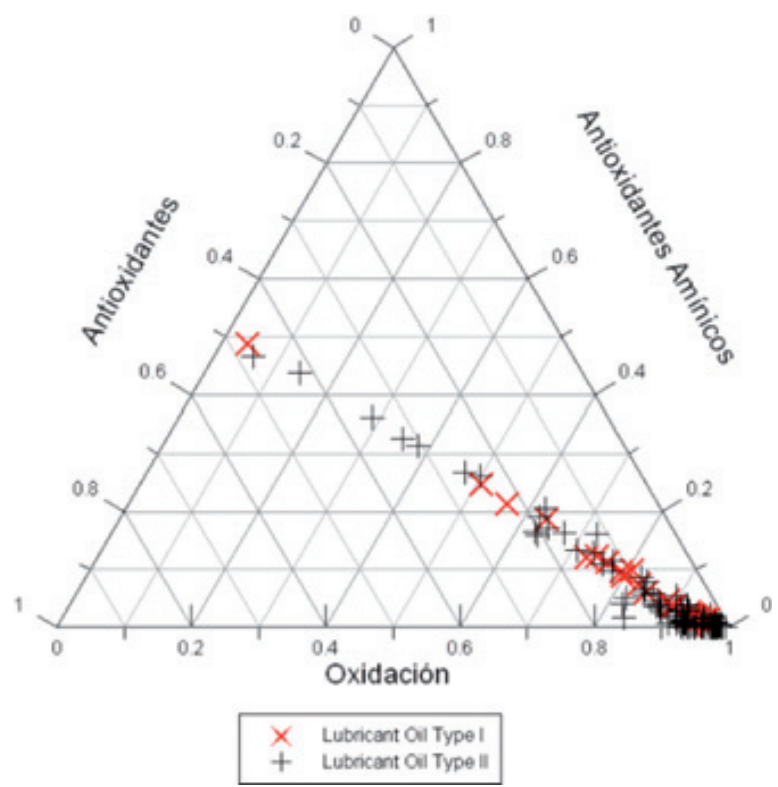

Fig. 17: Relación entre los niveles de oxidación, aditivos amínicos y antioxidantes

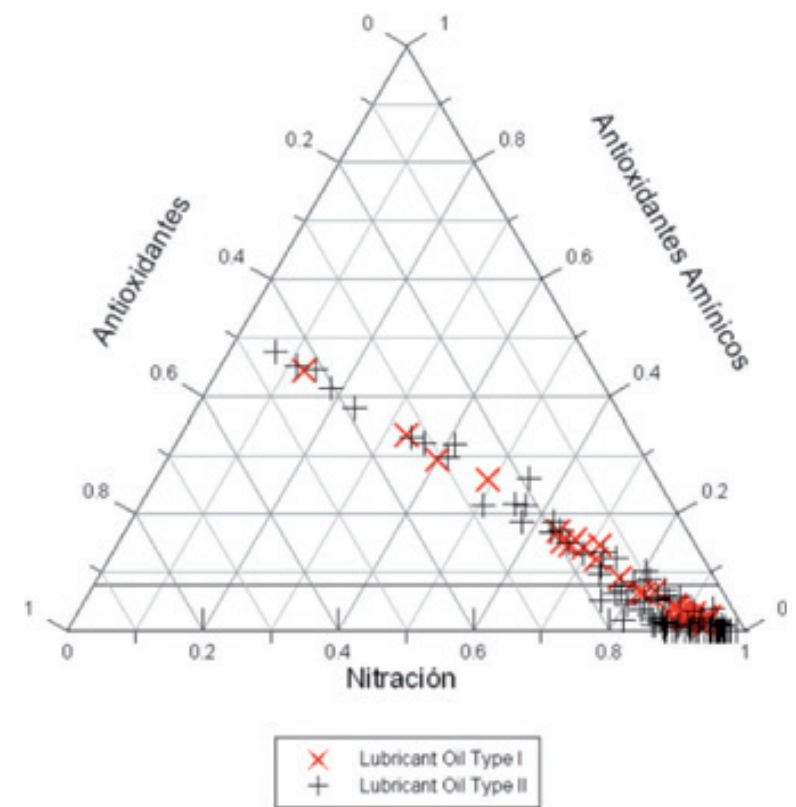

Fig. 18: Relación entre los niveles de nitración, aditivos amínicos y antioxidantes 
Los resultados que se han obtenido en motores GNC utilizando la formulación I, que presentan mejoras en su formulación (paquetes de aditivos y base lubricante), hace que estos puedan operar bajo condiciones más severas de servicio, permitiendo alcanzar los periodos de cambio aconsejados por los fabricantes sin poner en riesgo la vida del motor. A pesar de que el precio de la formulación I es aproximadamente 2,5 veces mayor que la formulación II, presenta mayores ventajas al cumplir con los periodos de servicio, disminuyendo los costes por inmovilizaciones y las operaciones de mantenimiento.

Se podría plantear la realización de algunas mejoras en los vehículos GNC con el fin de disminuir los altos niveles de estrés que estas configuraciones vienen presentando. Estas mejoras podrían estar enfocadas en cuanto a los volúmenes de aceite en el cárter o en cuanto a la eficiencia de los sistemas de refrigeración del aceite.

Existe una excelente correlación entre los paquetes de aditivos antioxidantes (aditivos antioxidantes y aditivos amínicos) y los niveles de oxidación y nitración, en todos los casos estudiados bajo condiciones reales de operación, como podemos ver en la figuras 17 y 18. Monitorizar una de estas variables (oxidación o nitración) a través de la técnica de FTIR y siguiendo la metodología empleada, permitirá llevar un excelente control de la degradación de los aceites lubricantes en MCIA.

\section{AGRADECIMIENTOS}

Los autores quieren mostrar su agradecimiento al apoyo recibido desde el Ministerio de Ciencia e Innovación - Dirección General de Investigación: TRA2008-06508 (GLAUTO).

\section{BIBLIOGRAFÍA}

[1] Shakour A, et al. "Natural gas as a promising alternative fuel for passenger cars". International Journal of Environment and Sustainable Development. Vol 2, (2) pp 184-196. 2003.

[2] Semin A, et al. "Combustion Temperature Effect of Diesel Engine Cover to Compressed Natural Gas Engine". American Journal. of Engineering and Applied Sciences 2 (1) pp 212-216. 2009.

[3] Adril E, et al. "Comparative Study of Characteristic of Lubricant Oils in Gasoline and Compressed Natural Gas Engines". European Journal of Scientific Research, 30 (2) pp 282-293. 2009.

[4] Macián V, et al. "Comparative Study of Engine Oil Performance on CNG/Diesel Engines on an Urban Transport Fleet". SAE Paper 2010-01-2100. DOI: http:// dx.doi.org/10.4271/2010-01-2100 . 2010.

[5] Macián V, et al. "Oil analysis evaluation for an engines fault diagnosis system". SAE Paper 1999-01-1515. DOI: http:// dx.doi.org/10.4271/1999-01-1515 . 1999.
[6] Macián V, et al. "Behavioural study of engine oil lubricants in gas engines used in urban transport fleets". Conference Proceedings, Lubrication Management and Technology LUBMAT '08. 2008.

[7] Dowson D, et al. "Life Cycle Tribology: 31st LeedsLyon Tribology Symposium". Tribology and Interface Engineering. Elsevier. 2005.

[8] Taylor R, et al. "Engine Lubricant Trends Since 1990". Engineering Tribology, pp 1-16. 2005.

[9] Coates J. "Interpretation of infrared spectra, a practical approach. Encyclopedia of Analytical Chemistry", John Wiley \& Sons Ltd, Chichester, pp. 10815-10837. 2000. DOI: http://dx.doi.org/10.1002/9780470027318.a5606

[10] Powell J et al. "Automated FT-IR Spectrometry for Monitoring Hydrocarbon-Based Engine Oils". Lubrication Engineering. 49, pp. 233-239. 1993.

[11] Van de Voort F, et al. "Monitoring the Oxidation of Edible Oils by Fourier Transform Infrared Spectroscopy". Journal of the American Oil Chemists' Society. 3, pp. 243-253. 1994.

[12] Van de Voort F, et al. "FT-IR Condition Monitoring of In-service lubricants: Ongoing Developments and Future Perspectives". Tribology Transactions. 49, pp.410-418. 2006.

[13] UNE-EN 590:2004/2010: "Combustibles para automoción. Combustibles para motor Diesel (gasóleo). Requisitos y métodos de ensayo."

[14] Kauffman R. "Remaining useful life measurements of Diesel engine oils, hydraulic fl uids and greases using cyclic voltammetric methods", Lubrication Engineering, Vol. 51, p. 223. 1994.

[15] Kauffman R. "Rapid, portable voltammetric techniques for performing antioxidant, total acid number (TAN) and Total base number (TBN) measurements", Lubrication Engineering, Vol. 54, p. 39. 1998.

[16] Kauffman R, et al "Development of a remaining useful life of a lubricant evaluation technique, part-I, differential scanning calorimetric techniques", Lubrication Engineering, Vol. 44, p. 154. 1988.

[17] Sharma B, et al "RUL assessment of lube oil using AHP and vector projection approach", Industrial Lubrication and Tribology, Vol. 58 Iss: 4, pp.187 - 194. 2006

[18] Rudnick L. Lubricant Additives - Chemistry and Applications. CRC Press Taylor \& Francis Group. 2003, ISBN-10: 0-8247-0857-1

[19] Macián V, et al. "Evaluation of used lubricant behaviour in industrial vehicles engines in the 1990s". SAE Paper 2000-01-1824. DOI: http:// dx.doi. org/10.4271/2000-01-1824 . 2000.

[20] Tormos B. "Contribución al diagnóstico de motores Diesel en basado en el análisis del lubricante usado". Tesis Doctoral. Universidad Politécnica de Valencia. 2002.

[21] Thibault R. "Converting to Condition-Based Oil Changes". Practicing Oil Analysis 2000 Conference Proceedings". 2000. 